\title{
Angular Momentum Surface Density of the Kerr Metric
}

\author{
L. Fernández-Jambrina and F. J. Chinea \\ Departamento de Física Teórica II, Facultad de Ciencias Físicas, Universidad Complutense 28040-Madrid, Spain
}

(Received 2 August 1993)

\begin{abstract}
A method for interpreting discontinuities of the twist potential of vacuum stationary axisymmetric solutions of Einstein's equations is introduced. Surface densities for the angular momentum of the source can be constructed after solving a linear partial differential equation with boundary conditions at infinity. This formalism is applied to the Kerr metric, obtaining a regularized version of the density calculated with other formalisms. The main result is that the integral defining the total angular momentum is finite for the Kerr metric.
\end{abstract}

PACS numbers: $04.20 . \mathrm{Cv}, 04.20 . \mathrm{Jb}, 97.60 . \mathrm{Lf}$

The physical interpretation of solutions of the Einstein equations is a task of great importance in general relativity. In the last few decades a great effort has been made to generate new solutions, but only a few have been studied from the physical point of view. In this Letter we provide a new method for constructing the angular momentum density of a source of a vacuum stationary axisymmetric solution of Einstein equations from the discontinuities of its twist potential. This is a generalization to curved spacetime of the potential theory formula for constructing dipole densities for a Newtonian field. As an example, the developed formalism is applied to the Kerr metric and the results are compared with those obtained by Israel in [1]. A brief discussion is provided.

We shall follow the formalism developed in $[2,3]$ for stationary axisymmetric perfect fluids and consider vacuum as a rigidly rotating perfect fluid whose four-velocity oneform $u$ is just the timelike leg of an orthonormal space- time vierbein. In canonical coordinates $(t, \phi, \rho, z)$, the metric is written

$$
\begin{aligned}
d s^{2}= & -e^{2 U}(d t+A d \phi)^{2} \\
& +e^{-2 U}\left[e^{2 k}\left(d \rho^{2}+d z^{2}\right)+\rho^{2} d \phi^{2}\right]
\end{aligned}
$$

and so we choose $u=e^{U}(d t+A d \phi)$ and define a form $w(w=* \omega$, where $\omega$ is the vorticity form associated to $u$ and $*$ denotes the two-dimensional Hodge dual in the space orthogonal to the orbits of the Killing vectors $\left.\left\{\partial_{t}, \partial_{\phi}\right\}\right)$ from

$$
d u=a \wedge u+w \wedge \theta^{1}
$$

where $\theta^{1}$ is a one-form orthonormal to $u$ in the space spanned by the orbits of the group of isometries, and $a$ is the acceleration form. We shall only consider metrics which are asymptotically flat. For our purposes we just need the following behavior at infinity in some coordinates $(t, r, \theta, \phi)$ :

$$
d s^{2}=-\left(1-\frac{2 m}{r}\right)\left(d t+\frac{2 J \sin ^{2} \theta}{r} d \phi\right)^{2}+\left(1+\frac{2 m}{r}\right)\left[d r^{2}+r^{2}\left(d \theta^{2}+\sin ^{2} \theta d \phi^{2}\right)\right]+O\left(1 / r^{2}\right),
$$

where $m$ is the total mass of the source and $J$ is the total angular momentum. From the Bianchi and Einstein vacuum equations, it follows $[2,3]$ that $w$ and $* w$ have to fulfill

$$
\begin{gathered}
d w+(b-2 a) \wedge w=0 \\
d * w+2 a \wedge * w=0
\end{gathered}
$$

where $b=d(\ln \rho)$. These two equations can be formally integrated:

$$
\begin{aligned}
& w=\rho^{-1} e^{2 U} d A, \\
& * w=e^{-2 U} d \chi
\end{aligned}
$$

where $\chi$ is the so-called twist potential and $A$ the met- ric function in (1). Einstein's equations for axisymmetric stationary vacuum metrics can be reduced to a complex second order partial differential equation for the Ernst potential [4], $\varepsilon=e^{2 U}+i \chi$. Our requirement about the asymptotic form of the metric imposes the following condition on $\chi$ :

$$
\chi=-2 J \cos \theta / r^{2}+O\left(1 / r^{3}\right) .
$$

We shall integrate over the whole space $V_{3}$ orthogonal to the velocity $u$ (whose metric is ${ }^{3} g=g+u \otimes u$ ) the projection of the gradient of a function $z$ (to be determined later) over the difference $e^{-U}[*(w)-* w]$, which will be obviously zero:

$$
\begin{aligned}
\sqrt{{ }^{3} g} e^{-U}\langle[*(w)-* w], d z\rangle & =\sqrt{{ }^{3} g} \partial_{\nu} z\left\{\rho^{-1} e^{U} \varepsilon^{\mu \nu} \partial_{\mu} A-e^{-3 U} g^{\mu \nu} \partial_{\mu} \chi\right\} \\
& =\partial_{\mu}\left([\mu \nu] A \partial_{\nu} z-\sqrt{{ }^{3} g} e^{-3 U} g^{\mu \nu} \chi \partial_{\nu} z\right)+\chi \partial_{\mu}\left(\sqrt{{ }^{3} g} e^{-3 U} g^{\mu \nu} \partial_{\nu} z\right)
\end{aligned}
$$

where $\varepsilon^{\mu \nu}=e^{2(U-k)}[\mu \nu]$ is the Levi-Cività tensor on the space orthogonal to the orbits of the Killing vectors and $\langle$, is the scalar product associated to the metric. We choose $z$ so that it satisfies 


$$
\partial_{\mu}\left(\sqrt{{ }^{3} g} e^{-3 U} g^{\mu \nu} \partial_{\nu} z\right)=0
$$

with the boundary condition $z=r \cos \theta$ at infinity. We assume now that the metric is continuous and that the twist potential is discontinuous across a closed surface $S$. This is no restriction since we can always close the surface taking the zero value for the discontinuity in the rest of it. The 3 -space $V_{3}$ is thus divided in two regions $V_{3}^{-}$(interior) and $V_{3}^{+}$(exterior) and we can apply Stokes' theorem to our integral:

$$
\begin{aligned}
0 & =\int_{V_{3}} \sqrt{{ }^{3} g} e^{-U}\langle[*(w)-* w], d z\rangle d x^{1} d x^{2} d x^{3} \\
& =\int_{\partial V_{3}^{+} \cup \partial V_{3}^{-}} n_{\mu} \partial_{\nu} z\left\{\rho^{-1} e^{U} \varepsilon^{\mu \nu} A-e^{-3 U} g^{\mu \nu} \chi\right\} d S .
\end{aligned}
$$

The boundary of $V_{3}^{+}$consists of $S$ and the sphere at infinity and the boundary of $V_{3}^{-}$is just $S$. According to our assumptions about asymptotic behavior and continuity, the integral at infinity can be computed to yield

$$
\int_{S} d S[\chi] e^{-3 U} g^{\mu \nu} n_{\mu} \partial_{\nu} z=-8 \pi J
$$

where we denote by $[\chi]$ the jump of the twist potential across $S$ and by $n$ the outward unit normal to $S$. From this formula we can interpret $-\frac{1}{8 \pi}[\chi] e^{-3 U} g^{\mu \nu} n_{\mu} \partial_{\nu} z$ as the angular momentum density of the two-dimensional source of the vacuum metric.

$$
\begin{aligned}
d s^{2}= & -\left(1-\frac{2 m r}{r^{2}+a^{2} \cos ^{2} \theta}\right)\left(d t+\frac{2 m a r \sin ^{2} \theta}{r^{2}+a^{2} \cos ^{2} \theta} d \phi\right)^{2} \\
& +\left(1-\frac{2 m r}{r^{2}+a^{2} \cos ^{2} \theta}\right)^{-1}\left\{\left(r^{2}-2 m r+a^{2}\right) \sin ^{2} \theta d \phi^{2}+\left(r^{2}-2 m r+a^{2} \cos ^{2} \theta\right)\left(\frac{d r^{2}}{r^{2}-2 m r+a^{2}}+d \theta^{2}\right)\right\}
\end{aligned}
$$

where $m$ is the total mass and $m a$, the total angular momentum. It fulfills the required asymptotic conditions for the previously described formalism to be applied. A solution for (10) with the prescribed boundary condition at infinity is

$$
z=(r-3 m) \cos \theta+\frac{2 a^{2} m\left(5 \cos ^{3} \theta-3 \cos \theta\right)}{5\left(r^{2}+a^{2} \cos ^{2} \theta\right)} .
$$

The disk $r=0$ has $n=\frac{1}{\cos \theta} \partial_{r}$ as a unit normal vector and the metric on this surface is

$$
d s^{2}=a^{2}\left(\cos ^{2} \theta d \theta^{2}+\sin ^{2} \theta d \phi^{2}\right) .
$$

Inserting these expressions in (12) we get the following expression for the angular momentum of the source:

$$
\begin{gathered}
J=\int_{0}^{2 \pi} d \phi \int_{0}^{\pi / 2} d \theta a^{2} \sin \theta \cos \theta \sigma_{J}, \\
\sigma_{J}=\frac{m}{2 \pi a \cos \theta} .
\end{gathered}
$$

Now we can apply this result to an example: The metric discovered by Kerr [5] is of great interest in astrophysics as the exterior of a rotating black hole. In 1970, Israel [1] used the theory of surface layers [6] to obtain the energy-momentum tensor of a minimal source for the Kerr metric that consisted of a disk. The mass and angular momentum densities obtained with this method are not integrable and therefore the singularity ring encircling the disk had to be considered. This was done by López applying the theory of distributions [7]. In what follows we shall calculate the angular momentum of the source taking into account the discontinuities of the twist potential.

The Ernst potential for the Kerr metric is $\varepsilon=1-$ $2 m /(r-i a \cos \theta)$ and so the twist potential is just $\chi=$ $-2 m a \cos \theta /\left(r^{2}+a^{2} \cos ^{2} \theta\right)$. This function takes the value $\chi(r=0)=-2 m \epsilon /(a \cos \theta), \theta \in[0, \pi / 2), \epsilon= \pm 1$, on approaching the disk $r=0$ from above or from below, respectively. This can be easily seen [1] considering pseudocylindrical coordinates:

$$
\begin{gathered}
P=\left(r^{2}+a^{2}\right)^{1 / 2} \sin \theta, \\
Z=r \cos \theta .
\end{gathered}
$$

The surface $r=0$ is then a disk of radius $P=a \sin \theta$ and therefore the points with polar angle $\theta$ and $\pi-\theta$ match on the disk. Hence, in what follows we shall restrict the range of $\theta$ to $[0, \pi / 2)$ on the disk $r=0$ to avoid double counting these points. The whole metric has the following expression:
The integral defining $J$ is perfectly regular and takes the value $m a$, which is consistent with the asymptotic expression of the metric.

The Ernst potential for the Kerr metric is a solution of the flat spacetime Laplace equation in oblate spheroidal coordinates [1]. Therefore its imaginary part can be viewed as a Newtonian potential and we can study its dipole density using potential theory [8]. As the disk of discontinuity is flat, the expressions obtained for $J$ and $\sigma_{J}$ in flat spacetime coincide with those obtained previously for the Kerr metric. Moreover, if we consider the classical potential that has $\sigma_{J}$ as a dipole source, it happens to be the same as the twist potential. That is, the twist potential for the Kerr metric, when considered as a Newtonian potential is completely generated by a dipole layer. No higher multipole layers need to be considered.

We can also compare the density $\sigma_{J}$ with the one obtained in [1]. In that reference, the calculated angular momentum density is $\sigma=-m \sin ^{2} \theta / 4 \pi a \cos ^{3} \theta$. This 
density is negative and nonintegrable and so it has to be compensated with an infinite angular momentum on the singularity ring. On the contrary, the density derived in this Letter is positive and integrable and so it could be viewed as a regularized version of [1]. A similar situation happens in magnetostatics, where the potential due to a ring of uniform current can be obtained also from a constant magnetic dipole density on the disk surrounded by the ring.

The present work has been supported in part by DGICYT Project PB89-0142; L.F.J. is supported by a FPI Predoctoral Scholarship from Ministerio de Educación y Ciencia (Spain). The authors wish to thank
L.M. González-Romero for valuable discussions.

[1] W. Israel, Phys. Rev. D 2, 641 (1970).

[2] F.J. Chinea and L.M. González-Romero, Classical Quantum Gravity 9, 1271 (1992).

[3] L.M. González-Romero, Ph.D. thesis, Universidad Complutense de Madrid, 1991 (unpublished).

[4] F.J. Ernst, Phys. Rev. 167, 1175 (1968).

[5] R.P. Kerr, Phys. Rev. Lett. 11, 237 (1963).

[6] V. de la Cruz and W. Israel, Phys. Rev. 170, 1187 (1968).

[7] C.A. López, Nuovo Cimento 66B, 17 (1981).

[8] O.D. Kellogg, Foundations of Potential Theory (Dover, New York, 1954). 